\title{
Fault detection in small diameter pipes using ultrasonic guided wave technology
}

\author{
Rahul M. Sabhnani ${ }^{a}$, Victor Humphrey ${ }^{b}$, Bahareh Zaghari ${ }^{b}$, Mohamed Moshrefi-Torbati $^{c}$ \\ ${ }^{a}$ School of Engineering Science, University of Southampton, Highfield, Southampton, SO17 \\ 1BJ, United Kingdom; \\ ${ }^{b}$ Institute of Sound and Vibration Research, University of Southampton, Highfield, \\ Southampton, SO17 1BJ, United Kingdom; \\ ${ }^{c}$ Electro-Mechanical Engineering Research Group, University of Southampton, Highfield, \\ Southampton, SO17 1BJ, United Kingdom;
}

\begin{abstract}
Ultrasonic guided wave technology is one of the more recent developments in the field of non-destructive evaluation. In contrast to conventional ultrasonic, this technology requires exposing only the areas where the transducers will be placed, hence requiring minimal insulation removal and excavation for buried pipes. This paper discusses how this technology can be used to detect defects in pipes under different conditions. Here the experiments were performed on small diameter pipes $(<5 \mathrm{~cm}$ diameter $)$; which were bare pipe, buried pipe and bitumen coated pipe. The results were gathered to see the effectiveness of this technology in detecting defects. Experiments were conducted using two dry coupled piezoelectric transducers, where one of them transmitted guided waves along the pipe and the other received them. The transducers produced tangential displacement, thereby generating the fundamental torsional mode $\mathrm{T}(0,1)$. In order to assess whether having multiple transducers has any effect on the resultant waveform, the receiving transducer was rotated around the circumference of the pipe.
\end{abstract}

Keywords: Bitumen, Buried pipe, Fault detection, Guided Wave, Non Destructive Testing, Piezoelectric, Pipes, Torsional mode, Transducer, Ultrasonic

\section{INTRODUCTION}

In this paper it is looked at how ultrasonic guided waves can be transmitted using a smart structure such as a piezoelectric transducer to detect defects in pipelines. As energy companies continue to spend billions in exploring and extracting fossil fuels; the role of pipelines and the safe delivery maybe neglected. Testing of pipelines without damaging them is crucial especially in the petrochemical industry to avoid leakages which can cause harm to all living beings. Pipelines deteriorate with time and to figure out the point of deterioration there is a need for a technology that is quick and accurate as some pipelines can be many kilometres long. Here it is discussed how ultrasonic guided waves, a Non-Destructive Testing (NDT) method, can be used to detect defects in different conditions. In contrast to many prior papers, focus was on performing experiments on small diameter pipes $(<5 \mathrm{~cm}$ diameter). The tests were performed using the torsional mode, $\mathrm{T}(0,1)$, to detect the defect located in the pipe and further tests were performed to understand how this technique responded when the pipe was coated with bitumen and when the pipe is buried in sand.

Further author information: (Send correspondence to Rahul M. Sabhnani)

Rahul M. Sabhnani: Email: rahul.sabhnani@harrispye.com, Telephone: +971508256311

Bahareh Zaghari: Email: B.Zaghari@soton.ac.uk, Telephone: +447842435451

Victor Humphrey: Email: vh@isvr.soton.ac.uk, Telephone: +442380594957

Mohammed Moshrefi-Torbati: Email: M.M.Torbati@soton.ac.uk, Telephone: +442380598583

Sensors and Smart Structures Technologies for Civil, Mechanical, and Aerospace Systems 2015,

edited by Jerome P. Lynch, Kon-Well Wang, Hoon Sohn, Proc. of SPIE Vol. 9435, 94353F

(c) 2015 SPIE - CCC code: 0277-786X/15/\$18 - doi: 10.1117/12.2085494

Proc. of SPIE Vol. $943594353 F-1$ 


\section{BACKGROUND}

\subsection{Ultrasonic Guided Wave Technology}

Ultrasonic guided wave is a non destructive method of testing which means it is possible to detect flaws, defects and corrosions on site. Guided waves was described by Izadpanah et al. ${ }^{1}$ as the exploitation of ultrasonics waves that propagate along a medium and are guided by geometric boundaries of the medium. The waves are generated by exciting a transducer with high voltage electric pulses; these pulses are converted to a mechanical vibration by a transducer. The waves then propagate through the material and are reflected back if there are any defects, welds or pipe ends along the path of the wave (see figure 1). The reflected waves are then received by the transducer and converted back to an electrical signal to be displayed on the oscilloscope. The received signals usually have higher amplitude than the background noise level. ${ }^{2}$

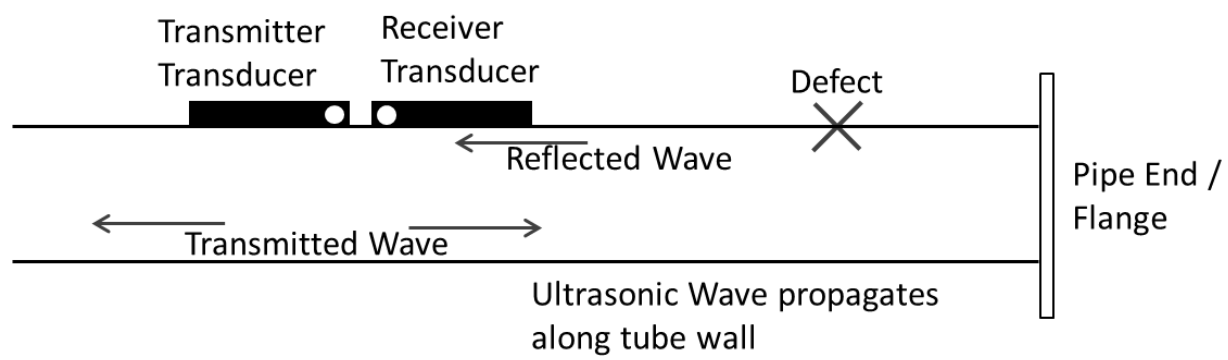

Figure 1. Transmission of guided waves along the pipe, with a piezoelectric transducer placed in each module. Here pulse echo transmission method had been adopted where the transmitting and receiving transducers were placed next to each other

One of the difficulties with guided waves is that their velocity is highly dependent on the wavelength and frequency, known as dispersion. Hence, dispersion curves are used to show the relations between the wave mode, velocities and frequency. Once the wave velocity and travel time are known, it can then be used to calculate the location of reflection i.e discontinuity within the pipe. When performing this testing there are two approaches that can be used - through transmission and pulse echo. For this experimentation pulse echo transmission was adopted as through transmission requires two opposing sides of the material being tested, since in reality companies prefer to perform tests on one side of the pipe, rather than to place the transmitting and receiving transducers far away from each other.

Ultrasonic guided waves are different to conventional ultrasonics which operates by exciting a wave along the thickness of the material (see figure 2). In the case of ultrasonic guided technology it propagates a wave along the axis of the pipe that travels long distances, which then is analysed in order to calculate the location of the defect. It also means that when using ultrasonic guided wave technology there is no need to excavate buried pipes hence making it cheaper to detect faults. The distance the guided waves can travel depends on the type of piping e.g. whether there is corrosion present or whether the pipe is coated or not. Table 1 shows the distance ultrasonic guided waves can travel based on the application.

\subsection{Types of guided waves in pipes}

Guided waves that propagate along a pipe can be generated by two means - axisymmetric and non-axisymmetric loading. Axisymmetric loading can produce two types of modes either longitudinal or torsional, while nonaxisymmetric loading produces flexural modes. As the name mentions axisymmetric waves are symmetric around the pipe axis, whereas non-axisymmetric waves are not. 


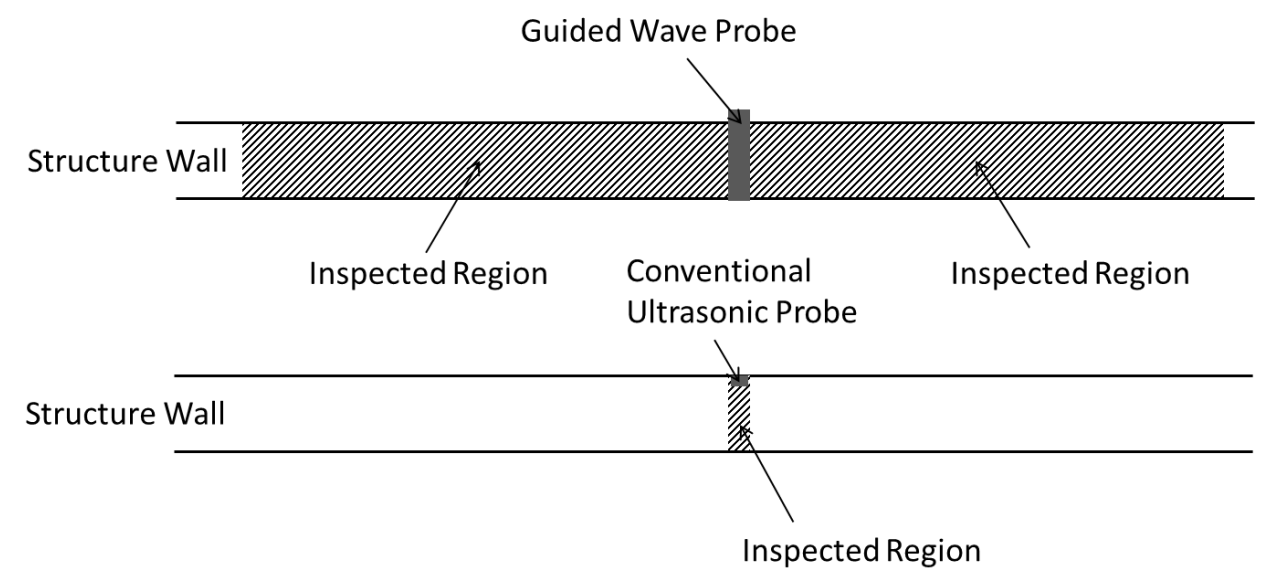

Figure 2. Propagation of waves using Ultrasonic Guided Wave Technology and conventional ultrasonic approach

Table 1. Typical range the guided waves propagate with a standard transducer ${ }^{3}$

\begin{tabular}{|l|c|}
\hline \multicolumn{1}{|c|}{ Application } & Range in each direction (m) \\
\hline Ideal Conditions & $80+$ \\
\hline 30 year old pipe with little internal and external corrosion & 40 \\
\hline 30 year old pipe with general corrosion & 20 \\
\hline Pipe wrapped in factory applied foam & 15 \\
\hline Heavily corroded pipe & 5 \\
\hline Bitumen coated pipe & $5^{*}$ \\
\hline \multicolumn{2}{|c|}{$*$ Range in bitumen coated pipe is dependent on bitumen condition } \\
\hline
\end{tabular}

Torsional mode involves a twisting motion where the displacement is in the theta direction. Longitudinal mode involves a compression motion where the displacement is in the radial and axial direction. Flexural mode involve a bending motion and these are more complicated as they can travel in either radial, axial or theta direction, see figure 3. Sonia and Fouad ${ }^{4}$ mentioned that axisymmetric modes are more often than not preferred over non-axisymmetric modes during experimentation as they are easier to excite and have relatively simpler acoustic fields. The naming of the waves is in accordance to Silk and Bainton ${ }^{5}$ where first letter is based on the type of wave - Longitudinal (L), Torsional $(\mathrm{T})$ or Flexural $(\mathrm{F})$. So for example $\mathrm{T}(\mathrm{n}, \mathrm{m})$ would be a torsional wave where ' $n$ ' is the harmonic order of circumferential variation (for axisymmetric modes this is 0 ) whereas ' $m$ ' represents mode and a fundamental mode is when ' $m$ ' $=1$.

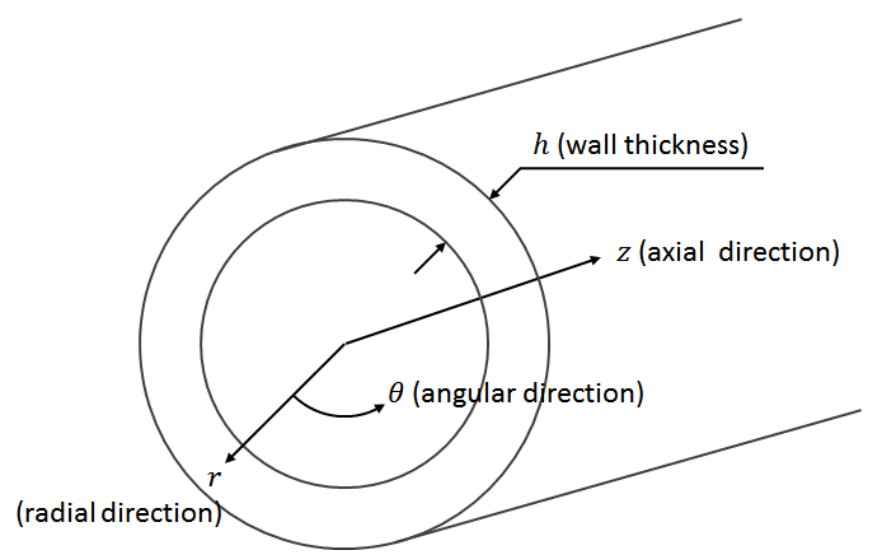

Figure 3. Schematic representation of pipe geometry 


\section{DEFECT DETECTION USING ULTRASONIC GUIDED WAVE}

\subsection{Experimental setup}

The experiments were conducted on a bare steel pipe with an outer diameter of $3.4 \mathrm{~cm}$ and a length of $280.3 \mathrm{~cm}$. The specimen had a notch that was machined $247 \mathrm{~cm}$ from the left end of the pipe. It was $5 \mathrm{~mm}$ in depth $(100 \%$ through the wall thickness) with an axial length of $4 \mathrm{~mm}$ and a circumferential length of $20 \mathrm{~mm}$ occupying $8.3 \%$ cross sectional area of the pipe wall, see figure 4 .

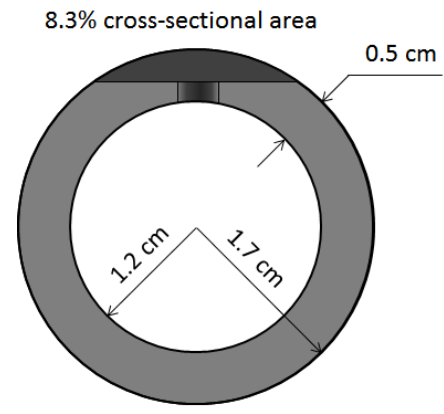

Figure 4. Schematic representation of the defect machined on the pipe

For all the experiments two dry coupled piezoelectric transducers were used, where one of them transmitted the guided waves along the pipe and other received the wave. The transducers produced tangential displacement, thereby generating the fundamental torsional mode $\mathrm{T}(0,1)$. The spacing between transducers and supporting equipments used during the experimentation can be seen in figure 5 .

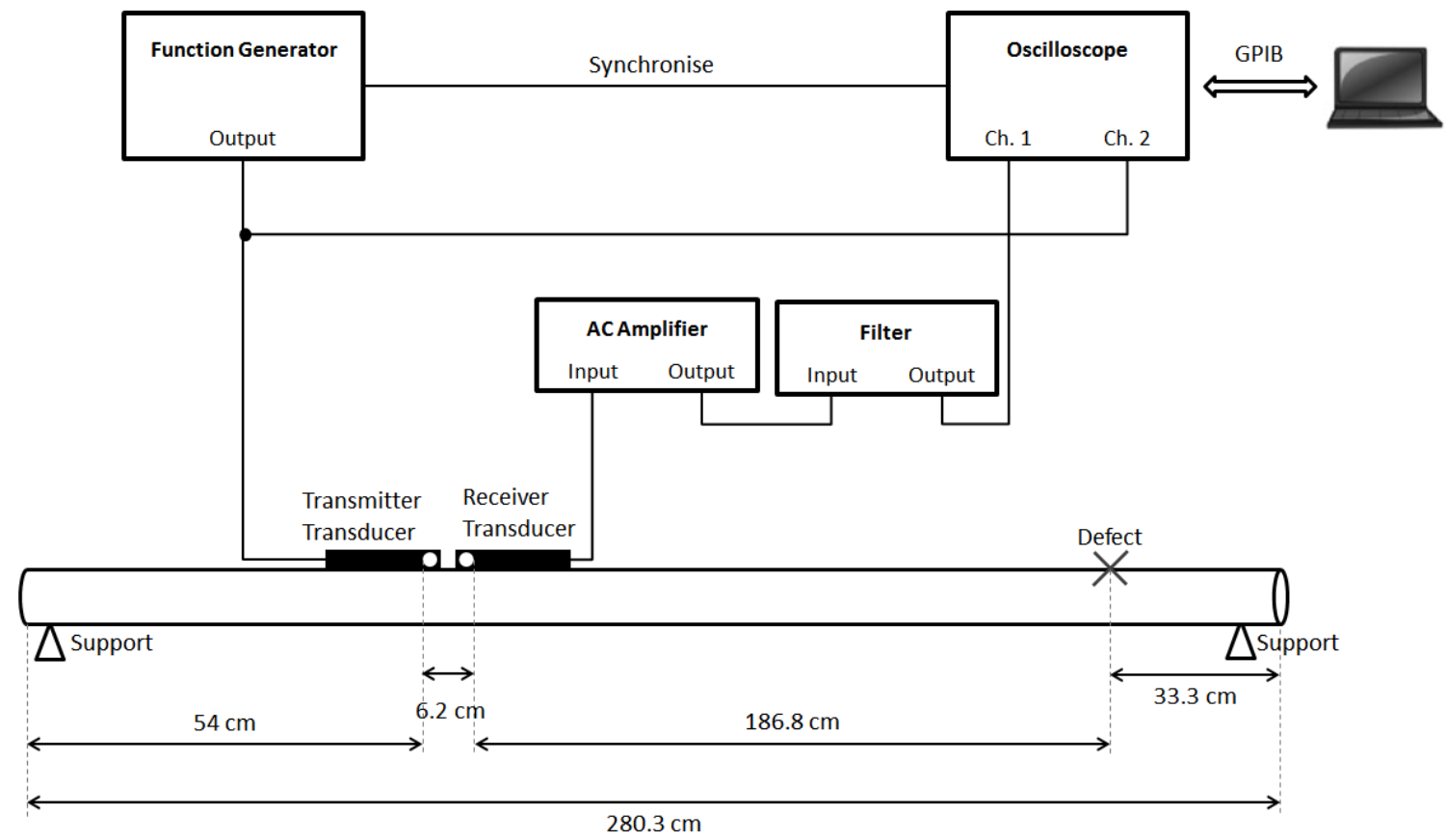

Figure 5. Experimental setup showing two transducers placed on the pipe along with the other connections 


\subsection{Single point measurement}

The aim of the experiment was to assess the theory mentioned by Demma et al. ${ }^{6}$ This theory stated that high frequency waves are more sensitive to small defects. The lowest frequency range used in practical testing with $\mathrm{T}(0,1)$ is $45-65 \mathrm{kHz}$ for a 3 inch pipe and $10-20 \mathrm{kHz}$ for 24 inch pipe. Demma et al. ${ }^{6}$ also mentions that a frequency range of $20-100 \mathrm{kHz}$ can be used to detect defects as small as $5 \%$ of the pipe's cross section area.

This experiment was performed by keeping the transducers in the same position i.e zero degree configuration which means that the transmitter and receiver were placed in line. To understand what feature each echo represents it is important to know the speed at which the waves propagate. A group velocity dispersion curve, figure 6 , was used to find the group velocity of the torsional mode, $\mathrm{T}(0,1)$. The velocity of $\mathrm{T}(0,1)$ mode can be estimated to be $3309 \mathrm{~ms}$

${ }^{-1}$. Using the velocity, the distances of defect and pipe end walls it was possible to estimate the arrival of each echo. Figure 7 shows the resultant waveform obtained from the guided wave transmitted at $50 \mathrm{kHz}$, it also shows the feature each echo represents.

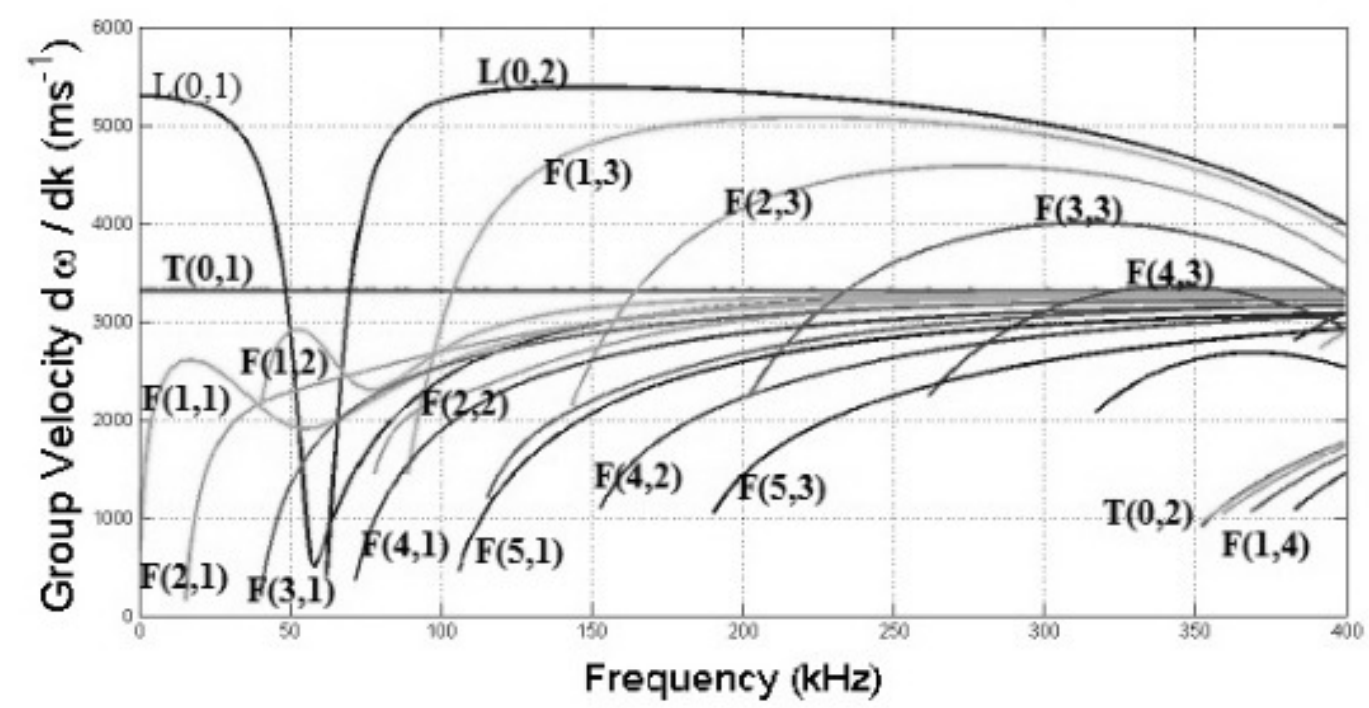

Figure 6. Group velocity dispersion curve, for steel pipe of outer diameter $34 \mathrm{~mm}$ and wall thickness $5.5 \mathrm{~mm}^{7}$

The point was to assess the theory of high frequency waves being more sensitive to small defects. Therefore guided waves were transmitted at a range of different frequencies ranging from $30 \mathrm{kHz}$ to $200 \mathrm{kHz}$, to assess how the defect echo varies and whether or not the defect echo was distinguishable from other echoes. It can be seen from figure 8 , that when guided waves are transmitted at $100 \mathrm{kHz}$ the defect echo is visible but it is difficult to distinguish and identify, whereas at frequency of $50 \mathrm{kHz}$ it is more clearly visible and distinguishable.

The reason for the defect echo becoming less visible when guided waves are transmitted at high frequencies lies in figure 6, which shows that as the frequency increases it propagates more modes. For example at $50 \mathrm{kHz}$ it has the fundamental torsional and longitudinal mode present along with the flexural modes $\mathrm{F}(1,1), \mathrm{F}(1,2), \mathrm{F}(2,1)$ and $\mathrm{F}(3,1)$. Likewise when the transmission frequency increases there are more non-axially symmetric modes that get excited as a result of which the received waveform displays signals from other modes that interfere with the defect signal. Thereby making the defect echo less easily identifiable and distinguishable. It should also be noted that these extra flexural modes that are generated are slower than the fundamental torsional mode, and hence these echoes appear later. 


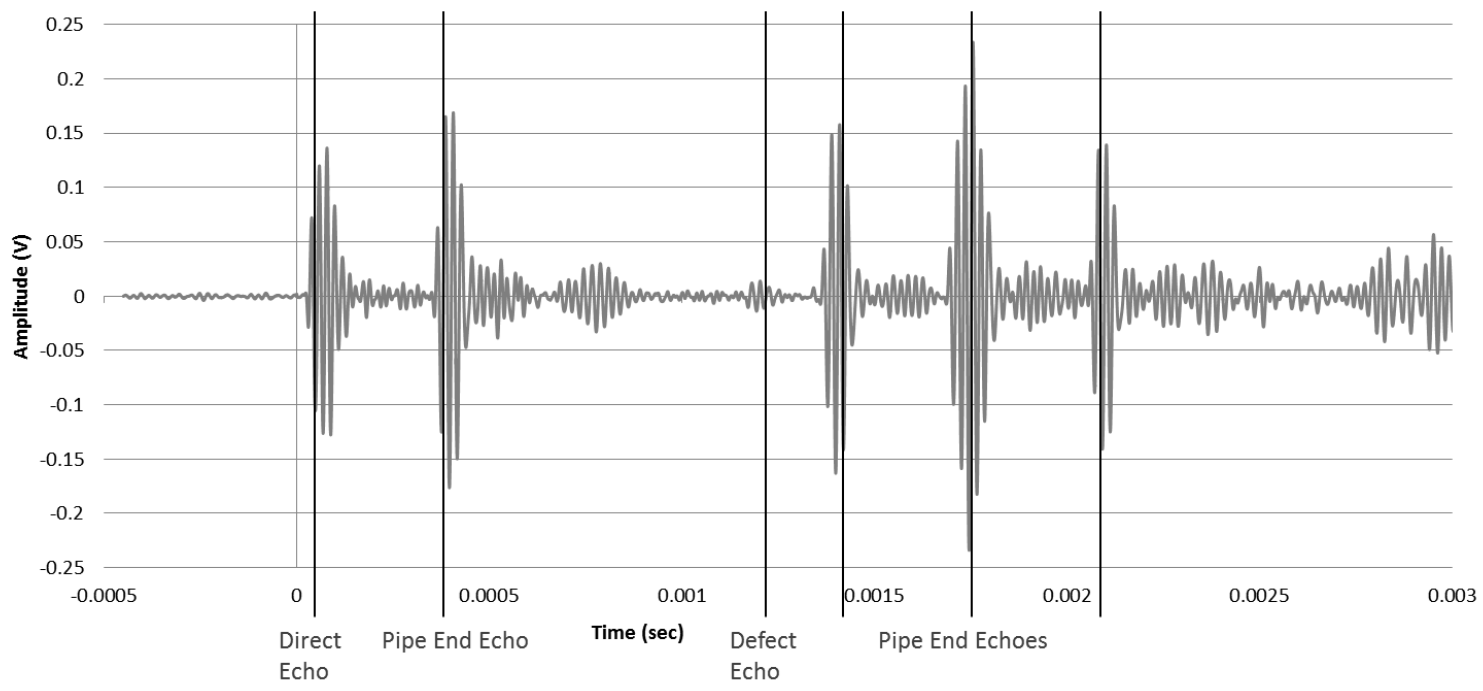

Figure 7. Amplitude of received signal for guided wave transmitted at $50 \mathrm{kHz}$

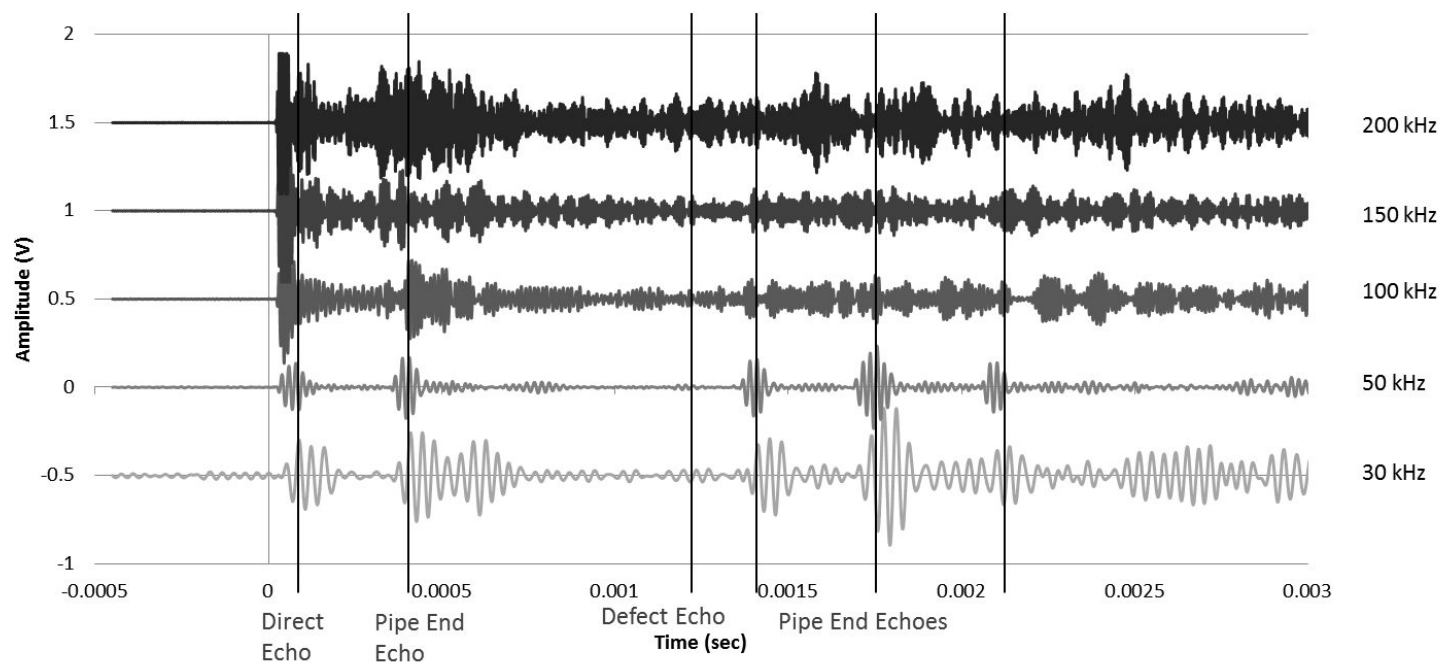

Figure 8. Amplitude of received signal for guided waves transmitted at different frequencies, when a single reading at zero degree configuration was taken 
Reverberation occurs due to reflection of part of the transmitted wave that is then received by the transducer, which makes it difficult to detect the defect. It occurs due to both axisymmetric and non-axisymmetric modes. Reverberation is different from noise. If noise was a limiting factor then the signal can be transmitted at higher level, thereby increasing transmission echo and not noise; but if reverberation is a limiting factor then increasing the signal will increase reverberation. Reverberation increases with frequency and it determines the frequency at which the guided wave can be transmitted. The frequency determines the smallest defect that can be detected by the guided wave. As mentioned earlier, it is known that guided waves transmitted at high frequencies are more sensitive to smaller defects than waves transmitted at low frequencies. The problem is that the defect echo gets lost in this reverberation, when the waves are transmitted at high frequencies using one transmitting and receiving transducer. In order to be able to clearly distinguish the defect echo, this reverberation must be reduced. This can be done by increasing the number of transducers used in the experiment.

\subsection{Multiple circumferential position measurements}

The aim of performing this experiment was to try to reduce the reverberation that occurs when the frequency of transmitted signal is increased. Alleyne and $\mathrm{Cawley}^{8}$, stated that it is possible to suppress the non-axisymmetric modes if an array of transducers are attached around the circumference of the pipe. This experiment was performed to test whether or not the theory is valid in practical applications, and if it reduces reverberation, thereby enabling ultrasonic signals to be transmitted at higher frequencies. Due to budget limitations it was not possible to purchase more transducers. Hence, it was decided that multiple readings will be taken around the circumference of the pipe and the resulting waveforms would be averaged.

The experimental setup was similar to that of the first experiment, except that in this case the receiving transducer was rotated around the circumference of the pipe whereas previously it was positioned at zero degree configuration. The receiving transducer was evenly spaced around the circumference of the pipe and readings taken before

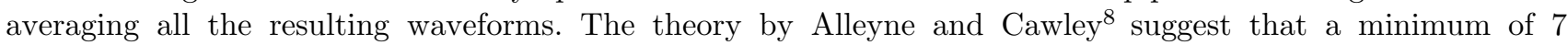
transducers are required around the circumference for a pipe in order to be able to able to suppress the flexural modes $\mathrm{F}(\mathrm{n}, \mathrm{m})$ when a wave is transmitted at $100 \mathrm{kHz}$ for a pipe with an outer diameter of $34 \mathrm{~mm}$. Their theory exploited the Nyquist-Shannon sampling theorem where the spacing between the transducers must be less than or equal to half of wavelength of the transmitted wave.

For this experiment 8 evenly spaced readings were taken around the circumference of the pipe and guided waves were transmitted at different frequencies ranging from $30 \mathrm{kHz}$ to $200 \mathrm{kHz}$. Figure 9 shows the 8 waveforms obtained when 8 readings were taken around the circumference, each separated by $45^{\circ}$. It also shows the average of 8 waveforms for guided waves transmitted at $100 \mathrm{kHz}$. This was similarly then performed for the other frequencies and the resultant average waveforms for each, with 8 readings, is shown in figure 10 .

From figure 10, it can be seen that the results have improved considerably compared to when only a single reading was taken in the first experiment (see figure 8). The pipe ends and the defect echoes are more clearly visible when multiple readings are taken around the circumference of the pipe and then averaged. This improvement is mainly due to the reduction in reverberation caused by non-axially symmetric modes along with the reduction in noise by averaging it out. There are some errors that might have occurred during the experimentation especially as there is a likelihood that the transducer was not placed in same place every time when performing experiment at different frequencies. Furthermore it was also experimented using 16 readings around the pipe, but there was not much of a reduction in reverberation, at $100 \mathrm{kHz}$. The only improvement was against noise which was minimal. The other method used was by placing the receiving transducer just below the transmitting transducer, which produced a lot of reverberation and was not successful (see figure 11). 

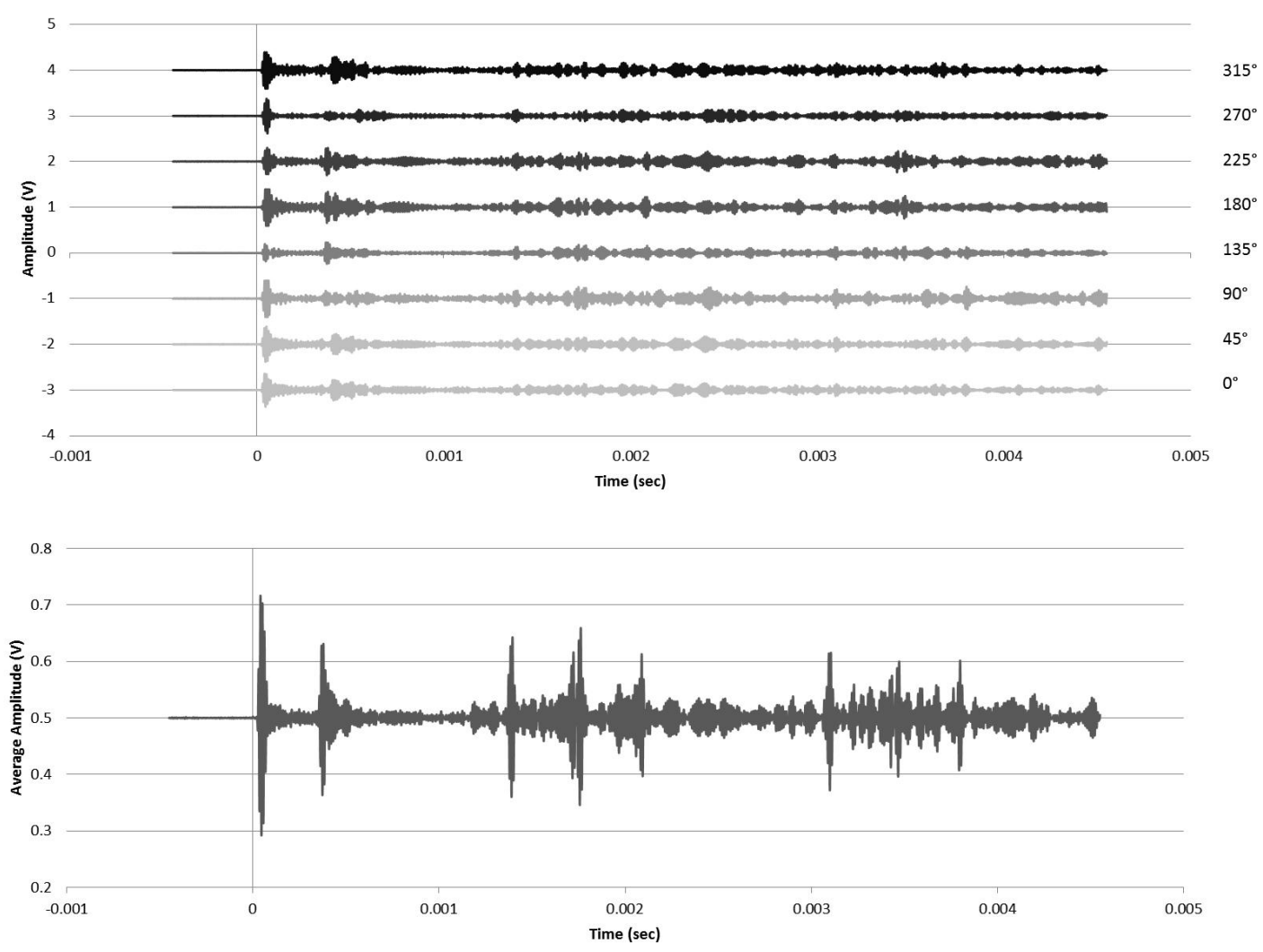

Figure 9. The top graph shows the received signals for 8 evenly spaced positions of the receiving transducer around the circumference of the pipe, for guided waves transmitted at $100 \mathrm{kHz}$, and the bottom graph shows the average signal of these 8 waveforms

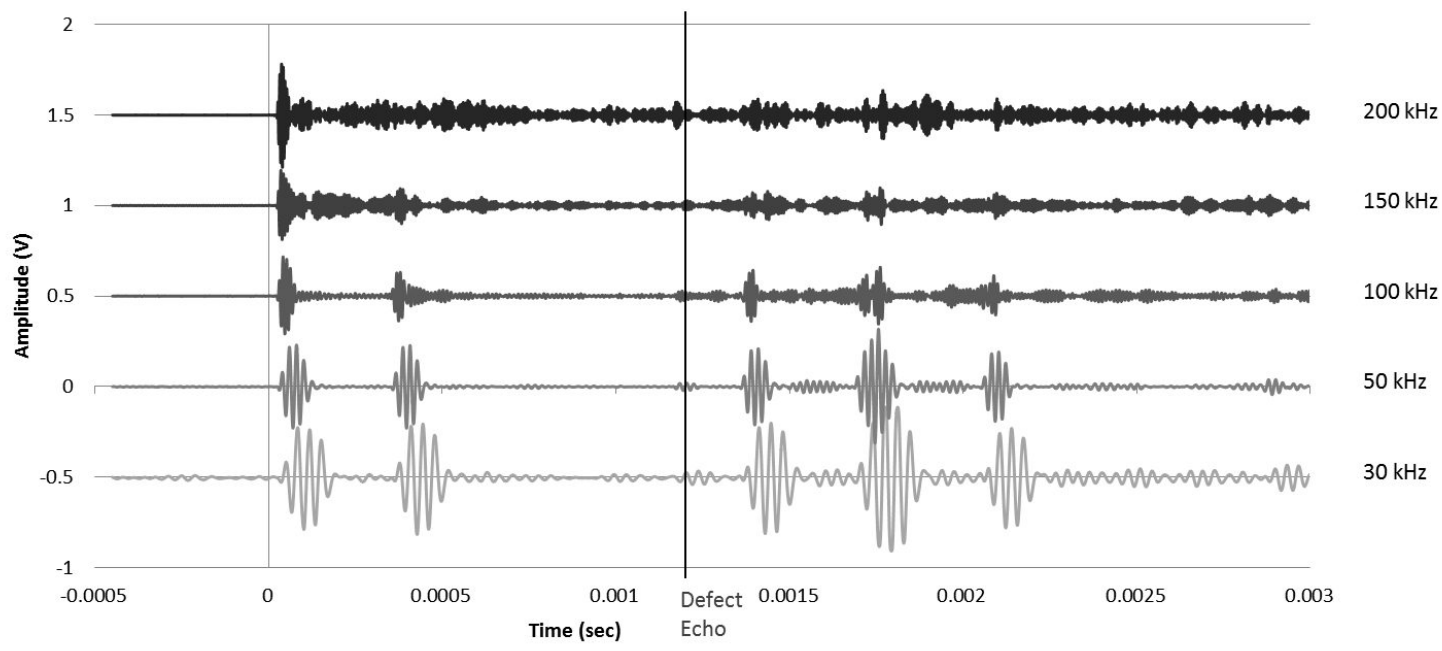

Figure 10. Average amplitude of the received signals, for guided waves transmitted at different frequencies, when 8 evenly spaced readings were taken circumferentially around the pipe 


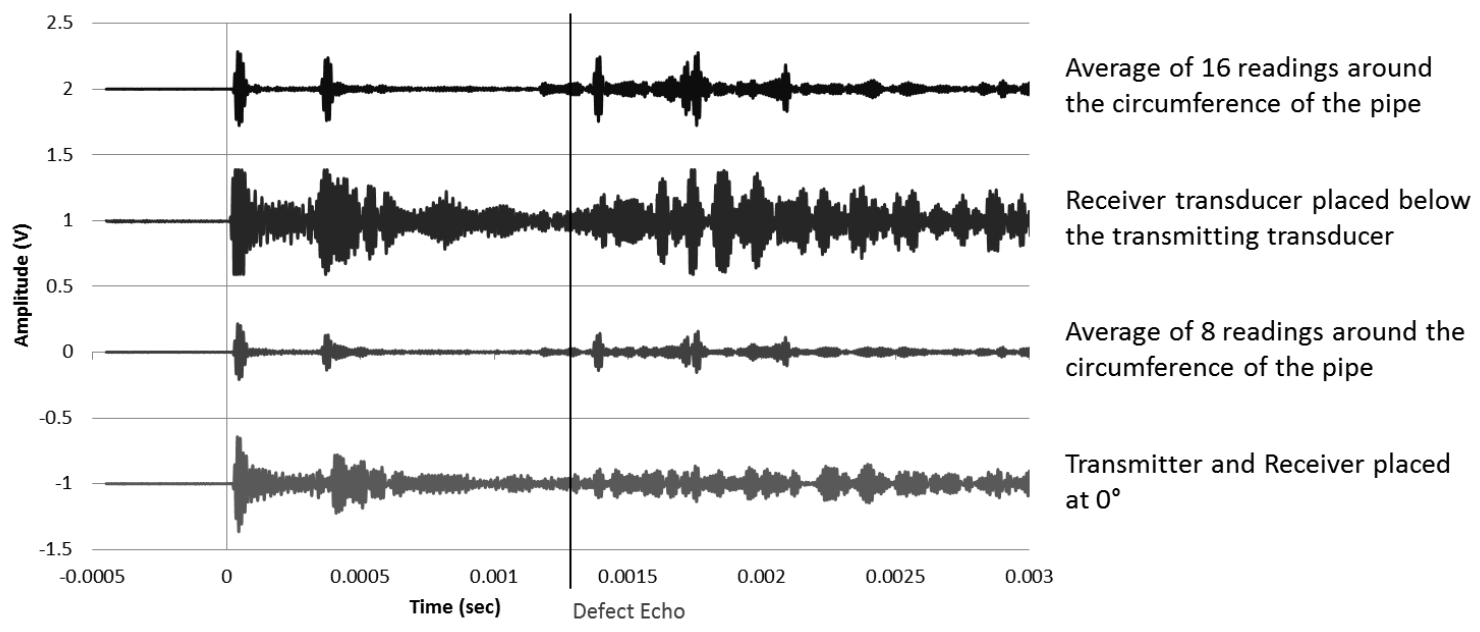

Figure 11. Comparing how resulting waveforms vary, for guided waves transmitted at $100 \mathrm{kHz}$, with variation in transducer positions. It shows, the output waveforms obtained when the transmitting and receiving transducer were placed at a zero degree configuration, when the receiving transducer was placed below the transmitting transducer, and average of 8 ( $45^{\circ}$ each) and $16\left(22.5^{\circ}\right.$ each) readings were taken around the circumference of the pipe

It should be noted that reverberation is caused by both axisymmetric and non-axisymmetric modes that get transmitted along with the fundamental torsional mode. Reverberation due to axisymmetric modes cannot be eliminated. Non-axisymmetric modes get eliminated because they have different phases at different positions, hence taking multiple readings and then averaging these readings results in a waveform with a lower reverberation. At higher frequencies there are more non-axisymmetric modes; hence more readings must be taken around the circumference of the pipe in order to have a lower reverberation. From this experiment it can be concluded that the theory stated by Alleyne and $\mathrm{Cawley}^{8}$, of using a series of elements around the circumference enables to compress the unwanted non-axisymmetric modes $\mathrm{F}(\mathrm{n}, \mathrm{m})$ and reduces the reverberation. This in turn means that it is possible to use higher frequencies to transmit the guided waves which would enable small defects to be detected.

\subsection{Buried pipe}

This experimentation was performed in order to assess if this technology can be used in industrial applications, where most of the pipelines are buried underground. For this experiment a box was made of plywood, through which the pipe was passed and then the box was filled with sand so that the defect was buried in the sand (approximately $50 \mathrm{~kg}$ of sand). Part of the pipe was left protruding out of the sand box where the transducers were placed. For this experimentation also 8 evenly spaced readings were taken around the circumference of the pipe and guides waves were transmitted for a range of frequencies from $30 \mathrm{kHz}$ to $200 \mathrm{kHz}$.

Figure 12, shows the resulting average waveforms obtained when 8 readings were taken around the circumference of the pipe for guided wave transmitted at a range of frequencies. As it can be seen from these waveforms they are very similar to the waveforms produced from the non-buried pipe with 8 readings (see figure 10).

The waveform produced from the experimentation on a buried pipe shows that there was minimal change in reverberation level for both the buried and non-buried pipe. It showed that the defect echo was visible at high frequencies as it can be detected at $50 \mathrm{kHz}$ and $100 \mathrm{kHz}$, though there was still high reverberation at 150 and 200 $\mathrm{kHz}$ which make it difficult to identify it. This result also showed that the sand does not bind to the pipe and hence there was no leakage of signal, although maybe different sand/soil types (e.g. clay, silt etc.) and the depth of soil could have varying effects. It has been shown by Rose et al. ${ }^{9}$, Ehrenberg and Torkelson ${ }^{10}$ that some soil types cause significantly high attenuation. Further research needs to be performed in different soil types before this technology can be utilised to its best effect in the industry. 


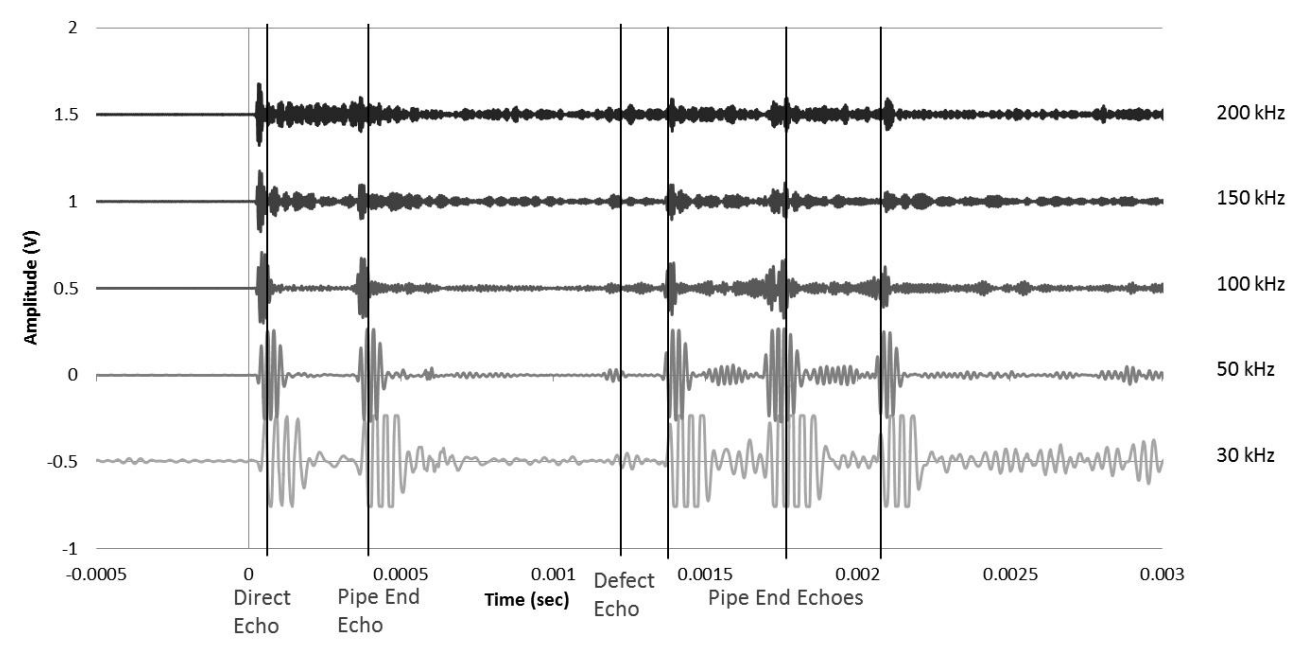

Figure 12. Average amplitude of received signals, for guided waves transmitted at different frequencies through the buried pipe, when eight evenly spaced readings were taken circumferentially around the pipe

\section{TRANSMISSION OF GUIDED WAVES THROUGH BITUMEN COATED PIPE}

\subsection{Experimental setup}

In section 2 and in table 1 it was mentioned that the one place where ultrasonic guided waves lack is their ability to travel long distance in bitumen coated pipes. In this experiment the aim was to understand the propagation of waves through the bitumen coated pipe and to see if the waves attenuate as strongly as mentioned in theory. Most of the old pipes are coated with bitumen and it is vital to know if this technology can be used to detect defects in these piping.

The experimental setup was similar to the ones used previously, with the fundamental torsional mode being used. The only difference in this case was the bare defect containing pipe was replaced with a bitumen coated pipe that contained no defects (see figure 13). In this experimentation only a single measurement was taken with the transmitting and receiving transducers placed at zero degree configuration; see figure 14 for the experimental setup. The bitumen coating was removed for the region where the transducers were placed in order to enable the transducers to couple onto the pipe.

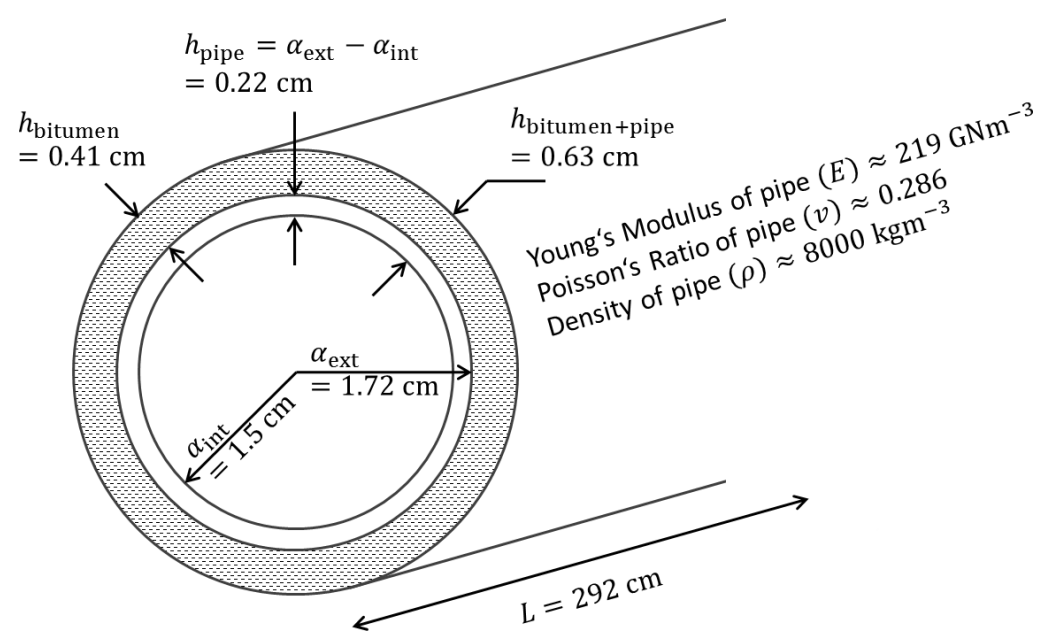

Figure 13. Dimensions and properties of the bitumen coated pipe 


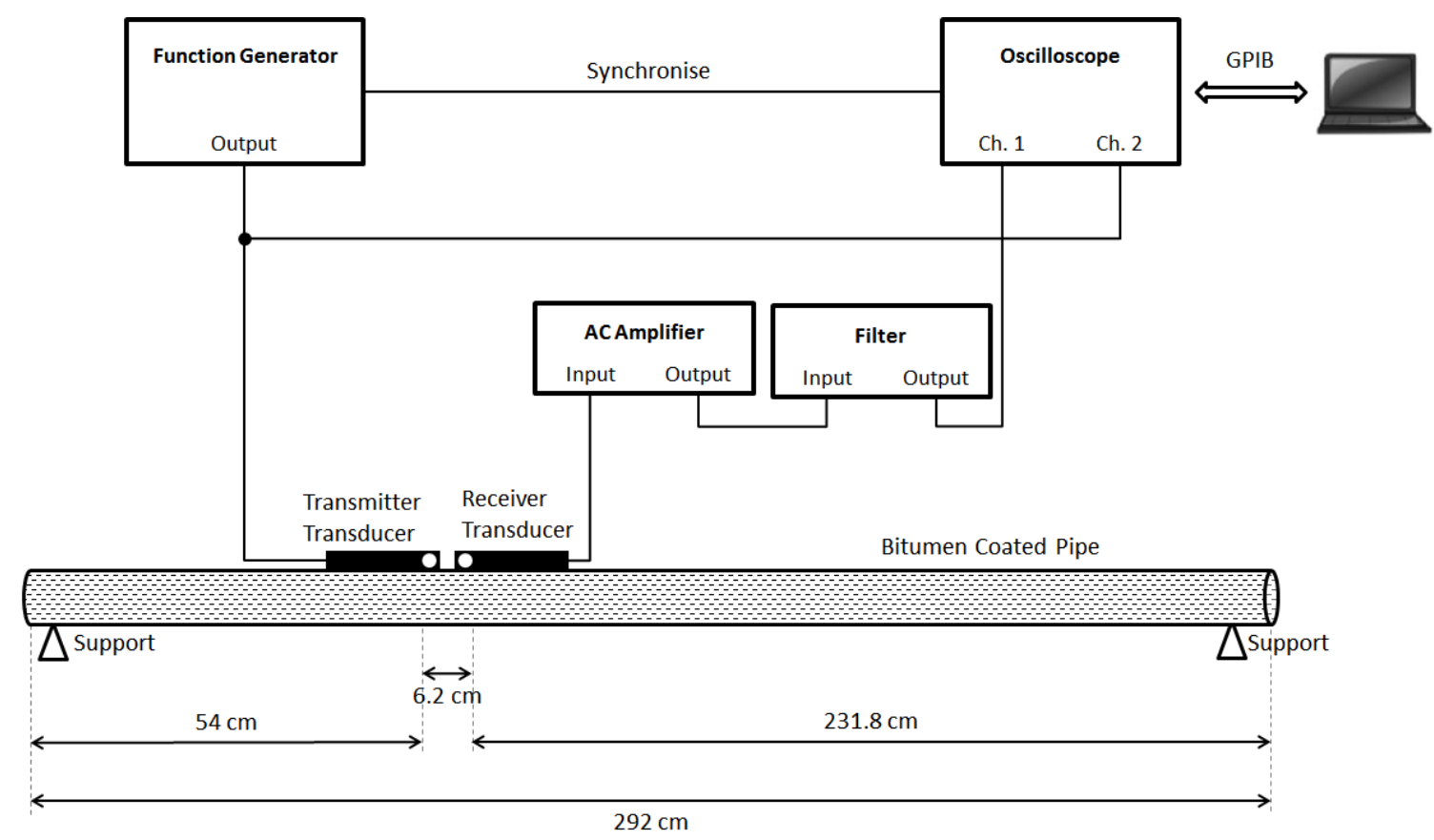

Figure 14. Experimental setup showing two transducers placed on the bitumen coated pipe. The transmitting and receiving transducers here were placed $6.2 \mathrm{~cm}$ apart from each other and the bitumen coating is removed in that area so that the transducers can couple onto the pipe

\subsection{Single point measurement}

As mentioned earlier that in this experiment there was no defect and the aim was to assess how far the ultrasonic guided waves can propagate before being absorbed by the bitumen coating. Similar to the previous experiments, guided waves were transmitted at different frequencies ranging from $30 \mathrm{kHz}$ to $200 \mathrm{kHz}$, but with only one reading taken at zero degree configuration. Figure 15 shows the waveforms obtained for guided waves transmitted at different frequencies.

It can be seen from the figure that only the first pipe end echo is visible and the attenuation is rather high as a result of which the guided waves dissipate quickly. At lower frequencies, i.e. $30 \mathrm{kHz}$ and to an extent $50 \mathrm{kHz}$, there was some aspects of pipe end echo visible, whereas at higher frequencies the only visible echo was due to direct transmission. This could be since low frequency waves travel further than high frequency waves.

Hence, from this experiment it was proven that the theory is correct and the ultrasonic guided waves attenuate strongly as a result of which the examination range reduces significantly for bitumen coated pipe. They perform worse than a bare buried pipe because the bitumen coating has a stronger bonding with the pipe compared to the sand. When pipes are coated with a viscoelastic material, such as bitumen, it causes the energy of ultrasonic guided waves to leak into the coated material from the pipe wall. The rate of leakage is determined by the strength of the coating. Bitumen being a strongly adhesive material binds firmly to the pipe, thereby resulting in leakage into the coating and causing significantly high attenuation of guided waves. This high attenuation, thereby results in limitation to the distance the guided waves can propagate. Lowe and P.Crawley ${ }^{11}$ mentioned that bitumen coated pipes attenuate guided waves as the pipe is a bi-layer system and any energy carried in bitumen layer is attenuated quickly, whereas in a corroded pipe the test range is lower as energy is scattered by the corroded surface. It is known from previous research that coatings that bind strongly with the pipe causes high attenuation whereas the ones that do not bind strongly such as mineral wool almost have no effect on the 


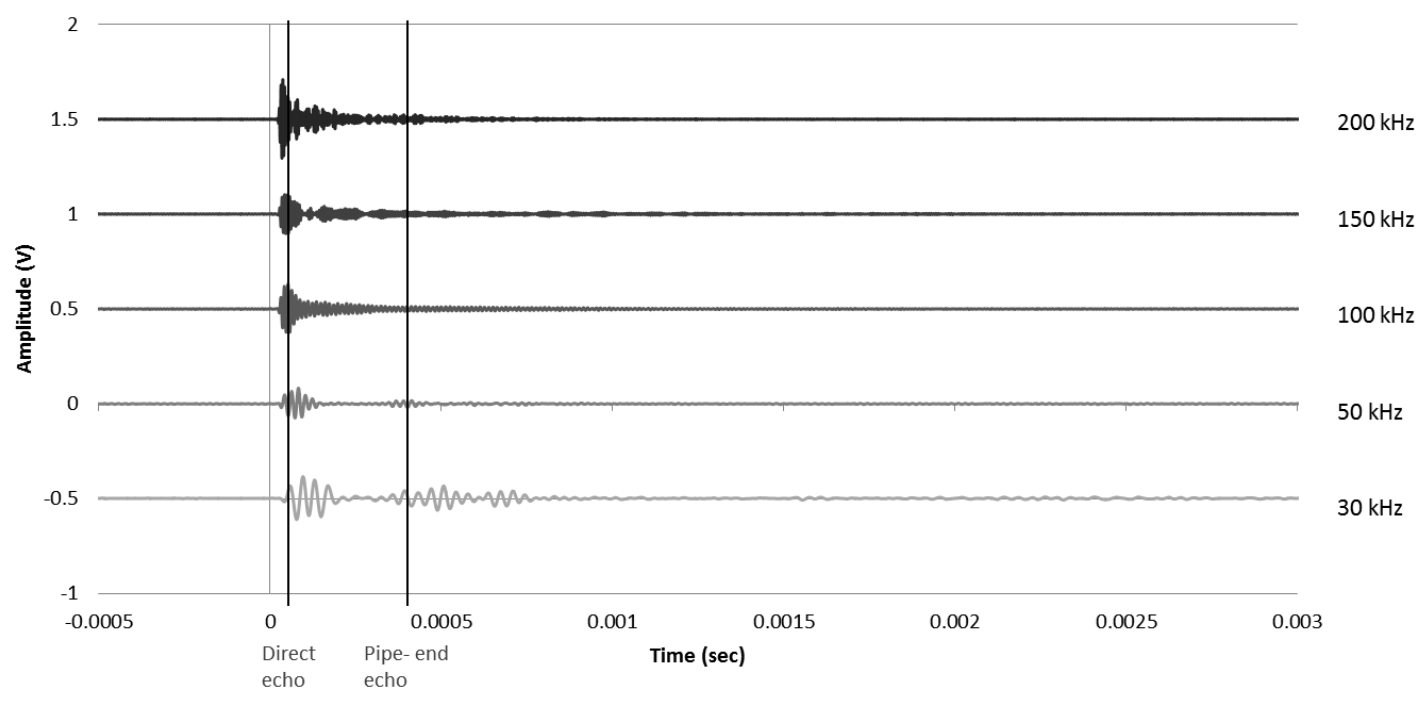

Figure 15. Amplitude of received signals for guided waves transmitted at different frequencies through a bitumen coated pipe, when a single reading at zero degree configuration was taken

waveform produced. In terms of coating it should be even remembered that the thickness and the age of coating also have an effect on attenuation. As can be seen from figure 15, when the frequency increases attenuation also increases which is because of the wavelength being shorter, therefore it dissipates easily into the bitumen coating. It is recommended that further research be performed on bitumen coated pipe with different thickness and age of coating along with trying to take multiple readings around the circumference of the pipe.

\section{CONCLUSION}

In this paper the suitability of ultrasonic guided wave technology was investigated in relation to small diameter pipes and experiments were performed on a steel pipe with a diameter of $34 \mathrm{~mm}$ and a wall thickness of 5 $\mathrm{mm}$. The first test was performed by taking a single measurement for guided waves transmitted at different frequencies. Here it was observed that as the frequency increases the reverberation level increases which makes it difficult to detect the echo, and high frequency waves are more sensitive to smaller defects therefore it is needed to transmit guided waves at higher frequencies.

Hence the next experimentation was performed by taking multiple readings around the circumference of the pipe. It was mentioned by Alleyne and Cawley ${ }^{8}$ that increasing the number of transmitters and receivers helps in suppressing the unwanted non-axisymmetric modes, which in turn would reduce reverberation. This proved to be successful as taking multiple readings did enable defect to be noticed when guided waves were transmitted at higher frequencies.

Next, two experiments were performed to assess whether this technology can be used for realistic application in industry. The first test was done on a bare pipe with defect buried in sand, by taking multiple readings around the pipe. This was performed to assess whether the technology can be used to detect defects for buried pipes. Results obtained for this experiment were very satisfactory as it was possible to identify the defect. Although it is necessary to perform further experiments on different soil types with varying depths. The second experiment was performed on a bitumen coated pipe as most of the old pipelines are bitumen coated and theory states that ultrasonic guided waves cannot travel long distance as they are absorbed by the bitumen. This was proven when the experiment was performed as the waves dissipated very quickly for guided waves transmitted over a range of frequencies. For future experiments it is recommended that different coatings be looked at along with different thickness and age of coating which can have an effect how far these waves can travel. It is also recommended that tests be performed on locally corroded pipe to assess if early corrosion can be detected. 


\section{ACKNOWLEDGEMENTS}

The authors would like to thank the laboratory technicians who helped us during the process of experimentation. The authors would like to thank the Institution of Mechanical Engineers (IMechE) for awarding Thomas Andrew Common Overseas Conference Grant in order to attend the conference.

\section{REFERENCES}

[1] Izadpanah, S., Rashed, G., and Sodagar, S., "Using ultrasonic guided waves in evaluation of pipes," in [The 2nd International Conference on Technical Inspection and NDT], (Oct. 2008).

[2] Gan, T. H., "The application of guided wave ultrasonic technique for the inspection of Corrosion Under Insulation (CUI) of pipes and pipelines," Petromin Pipeliner Magaziner , 32-37 (July-Sept. 2010).

[3] Webb, T. M., "Benefits and challenges of ultrasonic guided wave inspection," 2011 API Inspection Summit and Expo (2011).

[4] Sonia, D. and Fouad, B., "Propagation of guided waves in a hollow circular cylinder application to non destructive testing," in [14th International Conference on Experimental Mechanics], (June 2010).

[5] Silk, M. G. and Bainton, K. F., "The propagation in metal tubing of ultrasonic wave modes equivalent to lamb waves," Ultrasonic International 17(1), 11-19 (1979).

[6] Demma, A., Cawley, P., Lowe, M., and Roosenbrand, A. G., "The reflection of the fundamental torsional mode from cracks and notches in pipes," Journal of the Acoustical Society of America 114(2), 611-625 (2003).

[7] Zaghari, B., Humphrey, V., and Torbati, M. M., "Dispersion behaviour of torsional guided waves in small diameter steel gas pipes," in [19th International Conference on Automation 8 Computing, Brunel University], (September 2013).

[8] Alleyne, D. N. and Cawley, P., "The excitation of lamb waves in pipes using dry-coupled piezoelectric transducers," Journal of Nondestructive Evaluation 15(1), 11-20 (1996).

[9] Rose, J., Mu, J., Hua, J., Royer, R., and Kenefick, S., "Ultrasonic guided wave phased array inspection of coated and buried pipe (Part 2: experiment)," in [Developments in Ultrasonic Guided Wave Inspection], (June 2010).

[10] Ehrenberg, J. E. and Torkelson, T. C., "FM slide (chirp) signals: a technique for significantly improving the signal-to-noise performance in hydroacoustic assessment systems," Fisheries Research 47(2-3), 193-199 (2000).

[11] Lowe, M. and Crawley, P., "Long range guided wave inspection usage current commercial capabilities and research directions," tech. rep., Department of Mechanical Engineering Imperial College London, London UK (2006). 